\title{
Concise Syntheses of Cystothiazoles A, C, D, and Melithiazol B
}

\author{
Yuki IWAKI $^{a, b}$ and Hiroyuki AKITA ${ }^{*}, b$ \\ ${ }^{a}$ Research Institute, Novartis Pharma K.K.; 8 Ohkubo, Tsukuba, Ibaraki 300-2611, Japan: and ${ }^{b}$ School of Pharmaceutical \\ Sciences, Toho University; 2-2-1 Miyama, Funabashi, Chiba 274-8510, Japan. \\ Received July 4, 2007; accepted August 21, 2007; published online August 23, 2007
}

\begin{abstract}
A convergent synthesis of cystothiazoles $C 1$ and D 3 was achieved based on Julia coupling between the functionalized aldehyde 5b, corresponding to left half of the final molecule, and aryl sulfone 6 or 7 , bearing a bithiazole moiety, corresponding to right half. Methylation of 1 and 3 gave cystothiazole $A 2$ and melithiazol B 4, respectively. The overall yield (5 steps from $(2 R, 3 S)-3$-methylpent-4-yne-1,2-diol $10 ; 57 \%)$ of $5 \mathrm{~b}$ via the present route was improved in comparison to that of the previously reported functionalized aldehyde 5 a $(7$ steps from 10; $13 \%)$. By applying the modified Julia coupling method, selectivity $(6 E / 6 Z=20: 1-26: 1)$ toward the $(6 E)$-form of the coupled products $(15$ or 19$)$ against the corresponding $(6 Z)$-form was improved in comparison to the Wittig method $(6 E / 6 Z=4: 1-6.9: 1)$.
\end{abstract}

Key words cystothiazole; melithiazol; antibiotic; total synthesis; asymmetric synthesis; Julia coupling

Several antifungal substances, cystothiazoles A (2), C (1), $\mathrm{D}(\mathbf{3}))^{1,2)}$ and melithiazol B (4) ${ }^{3)}$ were isolated from different strains of the myxobacterium Cystobacter fuscus and Archangium gephyra, respectively. These antifungal substances possessing a bithiazole skeleton as well as a $\beta$ methoxyacrylate moiety, and $\mathbf{2}$ have shown potent antifungal activity against the phytopathogenic fungus Phytophthora capsici ( $2 \mu \mathrm{g} /$ disk), and have also shown activity against a broad range of additional fungi with no effect on bacterial growth. ${ }^{1}$ Furthermore, when $\mathbf{2}$ was examined for in vitro cytotoxicity using human colon carcinoma HCT-116 and human leukemia $\mathrm{K} 562$ cells, the resulting $\mathrm{IC}_{50}$ values were $110-130 \mathrm{ng} / \mathrm{ml}$, which were significantly higher than those of myxothiazol $\mathrm{A}^{4-6)}$ The fungicidal activity of these $\beta$ methoxyacrylate (MOA) inhibitors has been shown to be due to their ability to inhibit mitochondrial respiration by blocking electron transfer between cytochrome $b$ and cytochrome c. ${ }^{7}$ The absolute structure of $\mathbf{2}$ was established by a combination of spectroscopic analysis and chemical degradation of the natural product. ${ }^{1)}$ The left-side structure of $\mathbf{2}$ has been reported to be responsible for its antifungal activity. ${ }^{8)}$ For the purpose of studying the structure-biological activity relationships of cystothiazole A congeners, efficient synthesis of the left-side aldehyde 5a is considered to be highly important.

The first enantiocontrolled synthesis of $\mathbf{2}$ was achieved based on the preparation of a bis-thiazole core and application of an asymmetric Evans aldol methodology for development of the $C(4) / C(5)$ vicinal stereochemistry. ${ }^{9)}$ After our syntheses of cystothiazoles $A(\mathbf{2})^{10,11)}$ and $\mathrm{B}^{12)}$ were reported, further syntheses of cystothiazoles A $\mathbf{2}^{8,13-16)}$ and $\mathrm{B},{ }^{16)}$ as well as syntheses of the $C^{9,14)}$ and $E^{17,18)}$ types, were reported. In this paper, we describe a concise chiral synthesis of cystothiazoles A (2) and C (1) and melithiazol B (4) for the purpose of improving the overall yield and the $(E)$-selectivity at the $C(6) / C(7)$ double bond. Moreover, the first chiral synthesis of cystothiazole D (3) is described. Our retrosynthetic strategy for these natural products is illustrated in Chart 1. It can be seen that these compounds can be synthesized by modified Julia coupling ${ }^{19)}$ between the left-side aldehyde $\mathbf{5 b}$ and the right-side sulfone 6 or 7 . The synthesis of the leftside aldehyde $\mathbf{5 b}$ is shown in Chart 2.

The starting $(2 R, 3 S)$-diol $\mathbf{1 0}$ was obtained from $(2 R, 3 S)$ epoxy butanoate $\mathbf{8}^{20-23)}$ via $(2 R, 3 S)$-2-hydroxy ester 9 using a previously reported procedure. ${ }^{11)}$ Bis-silylation (11, quantitative yield) of $\mathbf{1 0}$ followed by consecutive treatment with $n$ BuLi and methyl chloroformate gave acetylenecarboxylate 12 in $93 \%$ overall yield. It was previously reported that conjugate addition of $\mathrm{MeOH}$ to a 3-substituted prop-2-ynoate congener in the presence of $\mathrm{Bu}_{3} \mathrm{P}$ selectively gave a $(2 E)-3-$ methoxy-acrylate congener. ${ }^{24)}$ Applying this procedure, conjugate addition of $\mathrm{MeOH}$ to acetylenecarboxylate $\mathbf{1 2}$ in the presence of a catalytic amount of $\mathrm{Bu}_{3} \mathrm{P}$ afforded a single isomer, $(E)$ - $\beta$-methoxy- $\alpha, \beta$-unsaturated ester 13 , in $90 \%$ yield. Determination of the $(E)$-geometry of $\mathbf{1 3}$ seemed to be difficult at this stage, but the geometry of $\mathbf{1 3}$ was confirmed by the fact that it was converted to the natural product cystothia-

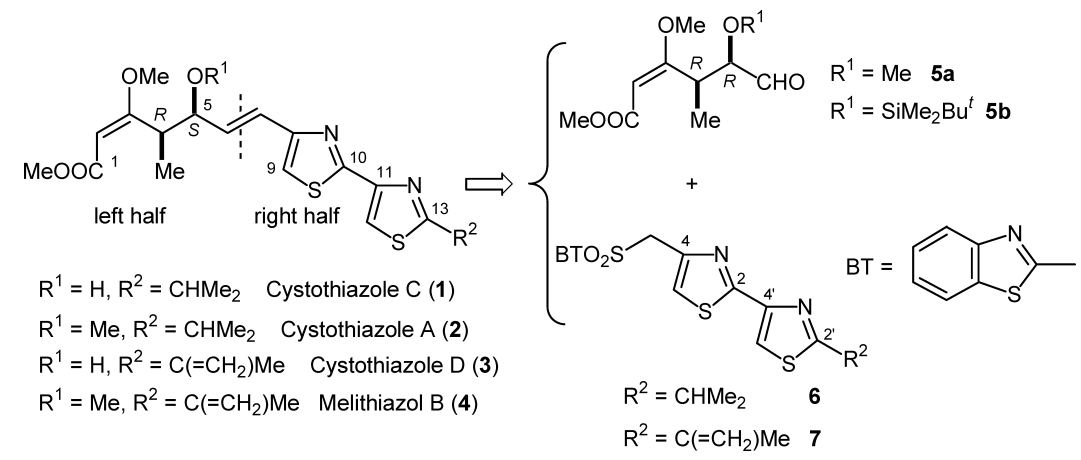

Chart 1 

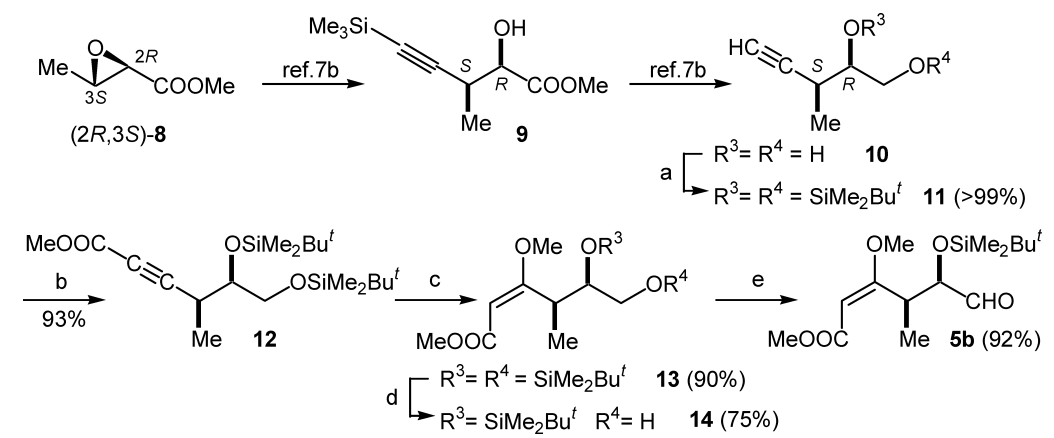

Reagent and conditions: (a) ${ }^{t} \mathrm{BuMe}_{2} \mathrm{SiCl} /$ imidazole/ $\mathrm{CH}_{2} \mathrm{Cl}_{2}$, (b) $n$ - $\mathrm{BuLi} / \mathrm{ClCOOMe} / \mathrm{THF}$, (c) $\mathrm{Bu} 3 \mathrm{P} / \mathrm{MeOH} / \mathrm{CH}_{2} \mathrm{Cl}_{2}$, (d) $\mathrm{HF} \cdot$ pyridine/THF, (e) Dess-Martin periodinane $/ \mathrm{CH}_{2} \mathrm{Cl}_{2}$.

Chart 2

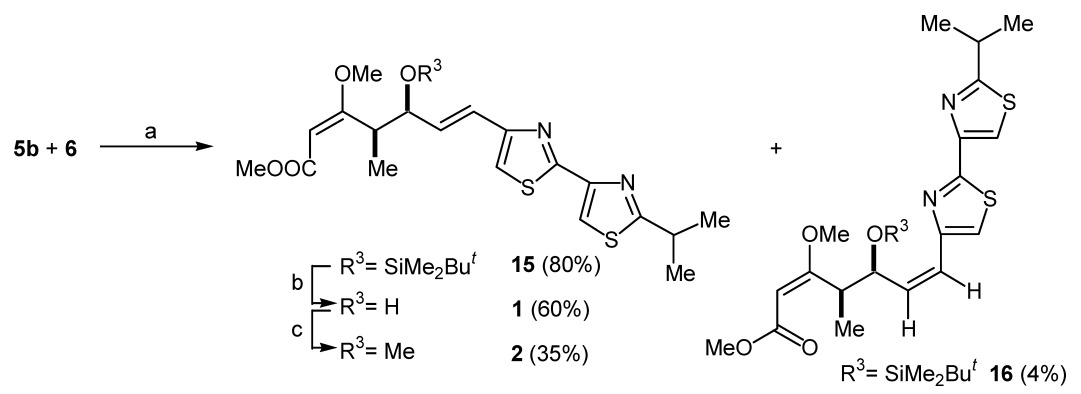

Reagents and conditions: (a) $\mathrm{LiN}\left(\mathrm{SiMe}_{3}\right)_{2} / \mathrm{THF}$, (b) $\mathrm{Bu}_{4} \mathrm{~F}^{+} \mathrm{F}^{-} / \mathrm{THF}$, (c) $\mathrm{Me}_{3} \mathrm{O}^{+} \mathrm{BF}_{4}^{-} /$proton sponge $/ \mathrm{CH}_{2} \mathrm{Cl}_{2}$.

Chart 3

zole C (1). Selective deprotection of the silyl groups in bissilyl ether 13 was achieved using HF - pyridine in THF to provide 14 in 75\% yield. Dess-Martin oxidation of 14 afforded the corresponding aldehyde $\mathbf{5 b}$ (92\% yield), which was used for the subsequent reaction without further purification. Next, modified Julia coupling of aldehyde $\mathbf{5 b}$ with the previously reported sulfone $6^{13)}$ was carried out (Chart 3 ). The reaction of $\mathbf{5 b}$ and $\mathbf{6}$ in the presence of lithium bis(trimethylsilyl)amide in THF gave a mixture $(E / Z=20 / 1)$ of coupled products, which were separated to give $(E)-15(80 \%)$ and (Z)-16 (4\%). Deprotection of $(E)-\mathbf{1 5}$ with tetrabutylammonium fluoride gave cystothiazole $C \quad \mathbf{1}\left([\alpha]_{\mathrm{D}}+142.7^{\circ}\right.$ $\left.\left(c=1.25, \mathrm{CHCl}_{3}\right)\right)$ in $60 \%$ yield. The spectral data $\left({ }^{1} \mathrm{H}-\right.$ and ${ }^{13} \mathrm{C}$-NMR) of synthetic 1 were identical to those of the natural product, ${ }^{2)}$ including the sign of specific rotation $\left([\alpha]_{\mathrm{D}}^{23}\right.$ $\left.+145^{\circ}\left(c=0.2, \mathrm{CHCl}_{3}\right)\right)$. Methylation of synthetic $1 \mathrm{using}$ Meerwein's reagent $\left(\mathrm{Me}_{3} \mathrm{O}^{+} \mathrm{BF}_{4}^{-}\right)$in the presence of proton sponge provided cystothiazole A $2\left([\alpha]_{\mathrm{D}}+105.2^{\circ}(c=0.34\right.$, $\left.\mathrm{CHCl}_{3}\right)$ ) in $35 \%$ yield along with the starting material 1 (37\% recovery). The spectral data $\left({ }^{1} \mathrm{H}-\right.$ and $\left.{ }^{13} \mathrm{C}-\mathrm{NMR}\right)$ of synthetic 2 were identical to those of the natural product, ${ }^{1)}$ including the sign of specific rotation $\left([\alpha]_{\mathrm{D}}+109^{\circ}(c=0.24\right.$, $\left.\mathrm{CHCl}_{3}\right)$ ).

Next the syntheses of cystothiazole D (3) and melithiazol B (4) were carried out (Chart 4). Treatment of the previously reported primary alcohol $17,{ }^{25)}$ bearing a bithiazole skeleton, with 2-mercaptobenzothiazole (BTSH) in the presence of $\mathrm{Ph}_{3} \mathrm{P}$ and diethylazodicarboxylate (DEAD) provided the sulfide 18 in $85 \%$ yield. This was then subjected to oxidation with $30 \% \mathrm{H}_{2} \mathrm{O}_{2}$ in the presence of $\mathrm{Mo}_{7} \mathrm{O}_{24}\left(\mathrm{NH}_{4}\right)_{6} \cdot 4 \mathrm{H}_{2} \mathrm{O}$ to give the corresponding sulfone 7 in $81 \%$ yield. The reaction of $\mathbf{5 b}$ and 7 in the presence of lithium bis(trimethylsilyl)- amide in THF gave a mixture $(E / Z=26 / 1)$ of coupled products, which were separated to give $(E)-19(51 \%)$ and $(Z)-20$ $(2 \%)$. Deprotection of $(E)-\mathbf{1 9}$ with tetrabutylammonium fluoride gave $3\left([\alpha]_{\mathrm{D}}+136.4^{\circ}\left(c=0.84, \mathrm{CHCl}_{3}\right)\right)$ in $53 \%$ yield. The spectral data $\left({ }^{1} \mathrm{H}\right.$ - and $\left.{ }^{13} \mathrm{C}-\mathrm{NMR}\right)$ of synthetic 3 were identical to those of the natural product, ${ }^{2)}$ including the sign of specific rotation $\left([\alpha]_{\mathrm{D}}+134^{\circ}\left(c=0.05, \mathrm{CHCl}_{3}\right)\right)$. Thus, the absolute structure of natural cystothiazole D (3) was confirmed by its first synthesis of 3. Methylation of synthetic 3 using Meerwein's reagent $\left(\mathrm{Me}_{3} \mathrm{O}^{+} \mathrm{BF}_{4}^{-}\right)$in the presence of proton sponge provided melithiazol B (4) $\left([\alpha]_{\mathrm{D}}+94.0^{\circ}\right.$ $\left.\left(c=0.72, \mathrm{CHCl}_{3}\right)\right)$ in $62 \%$ yield. The spectral data $\left({ }^{1} \mathrm{H}-\right.$ and ${ }^{13} \mathrm{C}$-NMR) of synthetic 4 were identical to those previously reported, $\left.{ }^{25}\right)$ including the sign of specific rotation $\left([\alpha]_{\mathrm{D}}^{23}\right.$ $\left.+83.6^{\circ}\left(c=0.99, \mathrm{CHCl}_{3}\right)\right)$.

In conclusion, convergent syntheses of cystothiazoles $\mathrm{C} \mathbf{1}$ and D 3 were achieved based on Julia coupling between the functionalized aldehyde $\mathbf{5} \mathbf{b}$, corresponding to left-side of the final molecule, and aryl sulfone 6 or 7 , bearing a bithiazole moiety corresponding to right-side. Methylation of $\mathbf{1}$ and $\mathbf{3}$ gave cystothiazole A (2) and melithiazol B (4), respectively. Bis-silylation of $(2 R, 3 S)$-3-methyl-pent-4-yne-1,2-diol 10, followed by introduction of a methoxycarbonyl group to the terminal acetylenic carbon, gave acetylenecarboxylate $\mathbf{1 2}$ (93\% overall yield), which was treated with $\mathrm{MeOH}$ in the presence of $\mathrm{Bu}_{3} \mathrm{P}$ to selectively afford $(E)$ - $\beta$-methoxyacrylate congener 13 in $90 \%$ yield. Selective desilylation of $\mathbf{1 3}$ gave primary alcohol 14 (75\%), which was subjected to Dess-Martin oxidation to afford the left-side aldehyde $\mathbf{5 b}$ (92\%). The overall yield ( 5 steps from 10; $57 \%$ ) of $\mathbf{5 b}$ via the present route was improved in comparison to that of 5a (7 steps from 10; 13\%) by way of the previously reported 


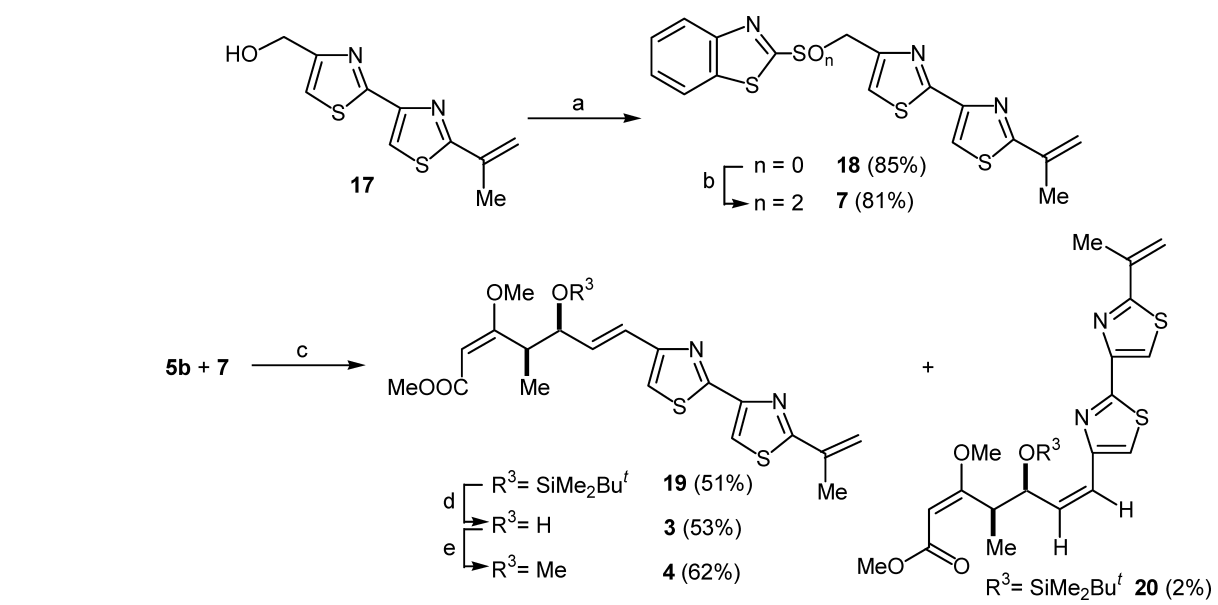

Reagents and conditions: (a) 2-mercaptobenzothiazole (BTSH)/DEAD/ $\mathrm{Ph}_{3} \mathrm{P} / \mathrm{THF}$, (b) $\mathrm{H}_{2} \mathrm{O}_{2} / \mathrm{Mo}_{7} \mathrm{O}_{24}\left(\mathrm{NH}_{4}\right)_{6} \cdot 4 \mathrm{H}_{2} \mathrm{O} / \mathrm{EtOH}$, (c) $\mathrm{LiN}\left(\mathrm{SiMe}_{3}\right)_{2} / \mathrm{THF}$, (d) $\mathrm{Bu}_{4} \mathrm{~N}^{+} \mathrm{F}^{-} / \mathrm{THF}$, (e) $\mathrm{Me}_{3} \mathrm{O}^{+} \mathrm{BF}_{4}^{-} /$proton sponge $/ \mathrm{CH}_{2} \mathrm{Cl}_{2}$.

Chart 4

route. ${ }^{10,11)}$ By applying this modified Julia coupling method, selectivity $(6 E / 6 Z=20: 1-26: 1)$ toward the $(6 E)$-form $(\mathbf{1 5}$ or 19) against the corresponding (6Z)-form was improved in comparison to the Wittig method $\left(6 E / 6 Z=4: 1^{11)}-6.9: 1^{15)}\right)$.

\section{Experimental}

General All melting points were measured on a Büchi 535 melting point apparatus and are uncorrected. ${ }^{1} \mathrm{H}$ - and ${ }^{13} \mathrm{C}-\mathrm{NMR}$ spectra were recorded on Bruker AV400M digital NMR spectrometer in $\mathrm{CDCl}_{3}$. High-resolution mass spectra (HR-MS) and the fast atom bombardment mass spectra (FAB-MS) were obtained with a JEOL JMS $600 \mathrm{H}$ spectrometer and JEOL GC-Mate spectrometer (matrix; $m$-nitrobenzylalcohol). IR spectra were recorded with a JASCO FT/IR-4100 spectrometer. Optical rotations were measured with a JASCO P-1020 digital polarimeter. All evaporations were performed under reduced pressure. For column chromatography, silica gel (KANTO silica-gel $60 \mathrm{~N}$, spherical, neutral, $40-50 \mu \mathrm{M}$ ) was employed.

$(-)-(3 S, 4 R)-4,5$-Bis-(tert-butyldimethylsilyloxy)-3-methylpent-1-yne (11) To a solution of (2R,3S)-3-methylpent-4-yne-1,2-diol $\quad(1.4 \mathrm{~g}$, $12.3 \mathrm{mmol})$ in $\mathrm{CH}_{2} \mathrm{Cl}_{2}(20 \mathrm{ml})$, imidazole $(2.09 \mathrm{~g}, 30.7 \mathrm{mmol})$ and tert-butyldimethylsilyl chloride $(4.62 \mathrm{~g}, 30.7 \mathrm{mmol})$ were added. The mixture was stirred at room temperature for $4 \mathrm{~h}$, and additional amounts of imidazole $(1 \mathrm{~g}, 14.7 \mathrm{mmol})$ and tert-butyldimethylsilyl chloride $(2 \mathrm{~g}, 13.3 \mathrm{mmol})$ were added with stirring for $2 \mathrm{~h}$ at $40^{\circ} \mathrm{C}$. The mixture was diluted with $\mathrm{CH}_{2} \mathrm{Cl}_{2}$, and washed with $\mathrm{H}_{2} \mathrm{O}$ and brine. The organic layer was dried $\left(\mathrm{Na}_{2} \mathrm{SO}_{4}\right)$, filtered, and concentrated in vacuo. The residue was purified by flash silica gel column chromatography $(70 \mathrm{~g}, n$-hexane/EtOAc $=9 / 1)$ to afford $\mathbf{1 1}(4.2 \mathrm{~g}$, quantitative yield) as a colorless oil. 11; $[\alpha]_{\mathrm{D}}^{22}-0.592^{\circ}\left(c=1.08, \mathrm{CHCl}_{3}\right)$, IR (neat): $3313,2116,1094 \mathrm{~cm}^{-1},{ }^{1} \mathrm{H}-\mathrm{NMR}\left(400 \mathrm{MHz}, \mathrm{CDCl}_{3}\right): \delta 0.08(6 \mathrm{H}, \mathrm{s})$, $0.10(6 \mathrm{H}, \mathrm{s}), 0.89(18 \mathrm{H}, \mathrm{s}), 1.16(3 \mathrm{H}, \mathrm{d}, J=7.1 \mathrm{~Hz}), 2.02(1 \mathrm{H}, \mathrm{d}, J=2.5 \mathrm{~Hz})$, $2.65-2.73(1 \mathrm{H}, \mathrm{m}), 3.57-3.63(2 \mathrm{H}, \mathrm{m}), 3.69(1 \mathrm{H}, \mathrm{dd}, J=10.6,5.3 \mathrm{~Hz})$ ${ }^{13} \mathrm{C}-\mathrm{NMR}\left(101 \mathrm{MHz}, \mathrm{CDCl}_{3}\right): \delta-5.40,-5.37,-4.66,-4.22,15.35,18.20$, 18.36, 25.91 (3C), 25.96 (3C), 28.72, 65.20, 69.00, 75.79, 87.72. HR-MS $(\mathrm{m} / \mathrm{z})$ : Calcd for $\mathrm{C}_{18} \mathrm{H}_{38} \mathrm{O}_{2} \mathrm{Si}_{2}: 285.1706(\mathrm{M}-t \mathrm{Bu})^{+}$. Found: 285.1705 .

$(4 S, 5 R)-5,6$-Bis-(tert-butyldimethylsilyloxy)-4-methylhex-2-ynoic Acid Methyl Ester (12) To a solution of $\mathbf{1 1}(3.9 \mathrm{~g}, 11.4 \mathrm{mmol})$ in THF $(40 \mathrm{ml})$ at $-78^{\circ} \mathrm{C}, 1.59 \mathrm{M}$ solution of $n$-butyllithium in $n$-hexane $(8.6 \mathrm{ml}, 13.6 \mathrm{mmol})$ was added. The mixture was stirred at $-78^{\circ} \mathrm{C}$ for $0.5 \mathrm{~h}$ and then methyl chloroformate $(1.06 \mathrm{ml}, 13.7 \mathrm{mmol})$ was added. After being warmed to room temperature and stirred for $1 \mathrm{~h}$, the reaction was quenched with aqueous saturated $\mathrm{NH}_{4} \mathrm{Cl}$ at $-78^{\circ} \mathrm{C}$. The mixture was diluted with EtOAc, the separated organic layer was washed with brine, dried $\left(\mathrm{Na}_{2} \mathrm{SO}_{4}\right)$, filtered and concentrated in vacuo. The residue was purified by flash silica gel column chromatography $(80 \mathrm{~g}, n$-hexane/EtOAc $=9 / 1)$ to afford $12(4.24 \mathrm{~g}, 93 \%)$ as a colorless oil. 12: $[\alpha]_{\mathrm{D}}^{24}-1.28^{\circ}\left(c=1.14, \mathrm{CHCl}_{3}\right)$, IR (neat): 2237,1720 $1254 \mathrm{~cm}^{-1},{ }^{1} \mathrm{H}-\mathrm{NMR}\left(400 \mathrm{MHz}, \mathrm{CDCl}_{3}\right): \delta 0.05(6 \mathrm{H}, \mathrm{s}), 0.08(3 \mathrm{H}, \mathrm{s}), 0.11$ $(3 \mathrm{H}, \mathrm{s}), 0.89(18 \mathrm{H}, \mathrm{s}), 1.19(3 \mathrm{H}, \mathrm{d}, J=7.0 \mathrm{~Hz}), 2.88(1 \mathrm{H}, \mathrm{dq}, J=7.0,4.5 \mathrm{~Hz})$ $3.52(1 \mathrm{H}, \mathrm{dd}, J=10.3,6.5 \mathrm{~Hz}), 3.58(1 \mathrm{H}, \mathrm{dd}, J=10.3,5.0 \mathrm{~Hz}), 3.75(3 \mathrm{H}, \mathrm{s})$, $3.76-3.80(1 \mathrm{H}, \mathrm{m}) .{ }^{13} \mathrm{C}-\mathrm{NMR}\left(101 \mathrm{MHz}, \mathrm{CDCl}_{3}\right): \delta-5.44,-5.38,-4.78$, $-4.29,13.64,18.17,18.33,25.86$ (3C), 25.94 (3C), 28.80, 52.51, 64.65,
73.52, 73.52, 74.68, 92.76, 154.30. Anal. Calcd for $\mathrm{C}_{20} \mathrm{H}_{40} \mathrm{O}_{4} \mathrm{Si}_{2} \cdot 1.5 \mathrm{H}_{2} \mathrm{O}: \mathrm{C}$, 58.63; H, 10.09. Found: C, 59.00; H, 10.11. HR-MS $(\mathrm{m} / \mathrm{z})$ : Calcd for $\mathrm{C}_{20} \mathrm{H}_{40} \mathrm{O}_{4} \mathrm{Si}_{2}: 343.1761\left(\mathrm{M}-{ }^{-} \mathrm{Bu}\right)^{+}$. Found: 343.1763 .

$(4 R, 5 R)$-5,6-Bis-(tert-butyldimethylsilyloxy)-3-methoxy-4-methylhex(2E)-enoic Acid Methyl Ester (13) To a solution of $12(0.95 \mathrm{~g}$, $2.37 \mathrm{mmol})$ in $\mathrm{CH}_{2} \mathrm{Cl}_{2}(5 \mathrm{ml})$ were added $\mathrm{MeOH}(0.48 \mathrm{ml})$ and tributylphosphine $(0.059 \mathrm{ml}, 0.24 \mathrm{mmol})$. The mixture was stirred at room temperature for $0.5 \mathrm{~h}$, and then concentrated in vacuo. The residue was purified by flash silica gel column chromatography $(30 \mathrm{~g}, n$-hexane/EtOAc $=9 / 1)$ to afford 13 $(0.93 \mathrm{~g}, 90 \%)$ as colorless oil. 13: $[\alpha]_{\mathrm{D}}^{23}-16.6^{\circ}\left(c=2.03, \mathrm{CHCl}_{3}\right)$, IR (neat): $1718,1631 \mathrm{~cm}^{-1},{ }^{1} \mathrm{H}-\mathrm{NMR}\left(400 \mathrm{MHz}, \mathrm{CDCl}_{3}\right): \delta 0.0099(3 \mathrm{H}, \mathrm{s}), 0.031(3 \mathrm{H}$, s), $0.049(3 \mathrm{H}, \mathrm{s}), 0.052(3 \mathrm{H}, \mathrm{s}), 0.87(9 \mathrm{H}, \mathrm{s}), 0.89(9 \mathrm{H}, \mathrm{s}), 1.03(3 \mathrm{H}, \mathrm{d}$, $J=6.8 \mathrm{~Hz}), 2.75(1 \mathrm{H}, \mathrm{dq}, J=6.8,3.8 \mathrm{~Hz}), 3.45(1 \mathrm{H}, \mathrm{dd}, J=7.3,10.1 \mathrm{~Hz})$, $3.52(1 \mathrm{H}, \mathrm{dd}, J=5.0,10.1 \mathrm{~Hz}), 3.66(3 \mathrm{H}, \mathrm{s}), 3.78-3.82(1 \mathrm{H}, \mathrm{m}), 3.88(3 \mathrm{H}$, s), $5.02(1 \mathrm{H}, \mathrm{s}) .{ }^{13} \mathrm{C}-\mathrm{NMR}\left(101 \mathrm{MHz}, \mathrm{CDCl}_{3}\right): \delta-5.46,-5.39,-4.94$, $-4.32,11.64,18.12,18.28,25.86$ (3C), 25.92 (3C), 39.86, 50.79, 59.21, 64.89, 73.43, 96.37, 165.93, 174.62. Anal. Calcd for $\mathrm{C}_{21} \mathrm{H}_{44} \mathrm{O}_{5} \mathrm{Si}_{2}: \mathrm{C}, 58.29$; $\mathrm{H}, 10.25$. Found: C, 57.82; H, 10.30. HR-MS $(\mathrm{m} / \mathrm{z})$ : Calcd for $\mathrm{C}_{21} \mathrm{H}_{44} \mathrm{O}_{5} \mathrm{Si}_{2}$ : $375.2023\left(\mathrm{M}-{ }^{t} \mathrm{Bu}\right)^{+}$. Found: 375.2033 .

$(4 R, 5 R)-5$-(tert-Butyldimethylsilyloxy)-6-hydroxy-3-methoxy-4methylhex-(2E)-enoic Acid Methyl Ester (14) To a solution of 13 $(0.152 \mathrm{~g}, 0.351 \mathrm{mmol})$ in THF $(1 \mathrm{ml})$ was added $\mathrm{HF} \cdot \mathrm{Py}(0.04 \mathrm{ml})$ at $0{ }^{\circ} \mathrm{C}$. The mixture was gradually warmed to room temperature, and then stirred for $6 \mathrm{~h}$. The reaction was quenched with saturated aqueous $\mathrm{NaHCO}_{3}$ at $0{ }^{\circ} \mathrm{C}$. The mixture was diluted with EtOAc and the separated organic layer was washed with brine, dried $\left(\mathrm{Na}_{2} \mathrm{SO}_{4}\right)$, filtered and concentrated. The residue was purified by flash silica gel column chromatography $(2 \mathrm{~g}, n$ hexane/EtOAc $=7 / 1)$ to afford $\mathbf{1 4}(0.084 \mathrm{~g}, 75 \%)$ as a colorless oil. 14: $[\alpha]_{\mathrm{D}}^{23}$ $+10.8^{\circ}\left(c=2.17, \mathrm{CHCl}_{3}\right)$, IR (neat): $3480,1716,1629 \mathrm{~cm}^{-1},{ }^{1} \mathrm{H}-\mathrm{NMR}$ $\left(400 \mathrm{MHz}, \mathrm{CDCl}_{3}\right): \delta 0.09(6 \mathrm{H}, \mathrm{s}), 0.91(9 \mathrm{H}, \mathrm{s}), 1.13(3 \mathrm{H}, \mathrm{d}, J=7.3 \mathrm{~Hz})$, $1.81(1 \mathrm{H}$, br. dd $J=6.08,6.08 \mathrm{~Hz}), 2.59(1 \mathrm{H}, \mathrm{dq}, J=7.3,7.08 \mathrm{~Hz}), 3.54$ $3.58(2 \mathrm{H}, \mathrm{m}), 3.66(3 \mathrm{H}, \mathrm{s}), 3.74(1 \mathrm{H}, \mathrm{ddd}, J=7.3,3.8,3.8 \mathrm{~Hz}), 3.94(3 \mathrm{H}, \mathrm{s})$, $5.05(1 \mathrm{H}, \mathrm{s}) .{ }^{13} \mathrm{C}-\mathrm{NMR}\left(101 \mathrm{MHz}, \mathrm{CDCl}_{3}\right): \delta-4.58,-4.29,15.22,18.17$, 25.91 (3C), 42.90, 51.02, 60.77, 64.75, 74.65, 95.91, 165.84, 173.83. HRMS $(\mathrm{m} / \mathrm{z})$ : Calcd for $\mathrm{C}_{15} \mathrm{H}_{30} \mathrm{O}_{5} \mathrm{Si}: 261.1158\left(\mathrm{M}-{ }^{t} \mathrm{Bu}\right)^{+}$. Found: 261.1156.

(4R,5R)-5-(tert-Butyldimethylsilyloxy)-3-methoxy-4-methyl-6-oxohex(2E)-enoic Acid Methyl Ester (5b) To a solution of $\mathbf{1 4}(0.210 \mathrm{~g}$, $0.659 \mathrm{mmol})$ in $\mathrm{CH}_{2} \mathrm{Cl}_{2}(2 \mathrm{ml})$ was added Dess-Martin periodinane $(0.308 \mathrm{~g}$, $0.726 \mathrm{mmol}$ ) at $0{ }^{\circ} \mathrm{C}$. After being stirred at room temperature for $0.5 \mathrm{~h}$, saturated aqueous $\mathrm{NaHCO}_{3}$ was added at $0{ }^{\circ} \mathrm{C}$. The organic layer was separated, washed with brine, dried $\left(\mathrm{Na}_{2} \mathrm{SO}_{4}\right)$, filtered and concentrated in vacuo. The residue was passed through short silica gel pad to afford $\mathbf{5 b}(0.192 \mathrm{~g}, 92 \%)$ as colorless oil. This product was used for the next reaction without further purification. Because this product was not stable, so it was promptly used to the next reaction. 5b: ${ }^{1} \mathrm{H}-\mathrm{NMR}\left(400 \mathrm{MHz}, \mathrm{CDCl}_{3}\right): \delta 0.05(6 \mathrm{H}, \mathrm{d}$, $J=5.8 \mathrm{~Hz}), 1.11(3 \mathrm{H}, \mathrm{d}, J=7.1 \mathrm{~Hz}), 2.70(1 \mathrm{H}, \mathrm{dq}, J=7.1,4.8 \mathrm{~Hz}), 3.67(3 \mathrm{H}$, s), $3.92(3 \mathrm{H}, \mathrm{s}), 4.05(1 \mathrm{H}, \mathrm{dd}, J=4.8,1.5 \mathrm{~Hz}), 5.06(1 \mathrm{H}, \mathrm{s}), 9.59(1 \mathrm{H}, \mathrm{d}$, $J=1.5 \mathrm{~Hz})$.

$(2 E, 6 E)-(4 R, 5 S)-5-($ tert-Butyldimethylsilyloxy)-7-(2'-isopropyl[2,4']bithiazolyl-4-yl)-3-methoxy-4-methylhepta-2,6-dienoic Acid Methyl 
Ester (15) and (2E,6Z)-(4R,5S)-5-(tert-Butyldimethylsilyloxy)-7-(2'-isopropyl-[2,4']bithiazolyl-4-yl)-3-methoxy-4-methylhepta-2,6-dienoic Acid Methyl Ester (16) To a solution of the reported aryl sulfone $6(0.281 \mathrm{~g}$, $0.60 \mathrm{mmol})$ in THF $(2 \mathrm{ml})$ was added $1.06 \mathrm{M}$ solution of lithium bis(trimethylsilylamide) in THF $(0.57 \mathrm{ml}, 0.61 \mathrm{mmol})$ at $-50{ }^{\circ} \mathrm{C}$. After being stirred at $-50^{\circ} \mathrm{C}$ for $0.5 \mathrm{~h}, \mathbf{5 b}(0.192 \mathrm{~g}, 0.607 \mathrm{mmol})$ in THF $(1 \mathrm{ml})$ was slowly added. The mixture was gradually warmed to room temperature and stirred for $0.5 \mathrm{~h}$. The reaction was quenched with saturated aqueous $\mathrm{NH}_{4} \mathrm{Cl}$ at $0{ }^{\circ} \mathrm{C}$. The mixture was extracted with EtOAc and washed with brine. The organic layer was dried $\left(\mathrm{Na}_{2} \mathrm{SO}_{4}\right)$, filtered and concentrated in vacuo. The residue was purified by flash silica gel column chromatography $(10 \mathrm{~g}, n$-hexane/EtOAc $=8 / 1)$ to afford $(6 Z)-16(0.014 \mathrm{~g}, 4 \%)$ and $(6 E)-15$ $(0.253 \mathrm{~g}, 80 \%)$ as colorless oil in elution order. $(6 E)-15$ : $[\alpha]_{\mathrm{D}}^{23}+79.1^{\circ}$ $\left(c=1.37, \mathrm{CHCl}_{3}\right)$, IR (neat): $3113,1711,1622 \mathrm{~cm}^{-1},{ }^{1} \mathrm{H}-\mathrm{NMR}(400 \mathrm{MHz}$, $\left.\mathrm{CDCl}_{3}\right): \delta 0.013(3 \mathrm{H}, \mathrm{s}), 0.034(3 \mathrm{H}, \mathrm{s}), 0.91(9 \mathrm{H}, \mathrm{s}), 1.16(3 \mathrm{H}, \mathrm{d}, J=$ $7.08 \mathrm{~Hz}), 1.43(3 \mathrm{H}, \mathrm{s}), 1.44(3 \mathrm{H}, \mathrm{s}), 3.34-3.41(1 \mathrm{H}, \mathrm{m}), 3.60(3 \mathrm{H}, \mathrm{s}), 3.65$ $(3 \mathrm{H}, \mathrm{s}), 4.03-4.09(1 \mathrm{H}, \mathrm{m}), 4.39-4.42(1 \mathrm{H}, \mathrm{m}), 4.95(1 \mathrm{H}, \mathrm{s}), 6.49-6.58$ $(2 \mathrm{H}, \mathrm{m}), 7.04(1 \mathrm{H}, \mathrm{s}), 7.84(1 \mathrm{H}, \mathrm{s}) \cdot{ }^{13} \mathrm{C}-\mathrm{NMR}\left(101 \mathrm{MHz}, \mathrm{CDCl}_{3}\right): \delta-4.96$, $-4.07,13.17,18.19,23.16(2 \mathrm{C}), 25.86(3 \mathrm{C}), 33.35,41.68,50.73,55.37$, $75.59,90.61,114.46,114.71,122.97,134.88,148.85,154.86,162.39$, 167.84, 177.70, 178.54, FAB-HR-MS $(m / z)$ : Calcd for $\mathrm{C}_{25} \mathrm{H}_{30} \mathrm{~N}_{2} \mathrm{O}_{4} \mathrm{~S}_{2} \mathrm{Si}$ 523.2121 $(\mathrm{M}+\mathrm{H})^{+}$. Found: 523.2121. (6Z)-16: $[\alpha]_{\mathrm{D}}^{22}+15.6^{\circ}(c=1.10$, $\mathrm{CHCl}_{3}$ ). IR (neat) $3109,1714,1631 \mathrm{~cm}^{-1},{ }^{1} \mathrm{H}-\mathrm{NMR}\left(400 \mathrm{MHz}, \mathrm{CDCl}_{3}\right): \delta$ $0.032(3 \mathrm{H}, \mathrm{s}), 0.062(3 \mathrm{H}, \mathrm{s}), 0.93(9 \mathrm{H}, \mathrm{s}), 1.14(3 \mathrm{H}, \mathrm{d}, J=7.06 \mathrm{~Hz}), 1.43$ $(3 \mathrm{H}, \mathrm{s}), 1.45(3 \mathrm{H}, \mathrm{s}), 2.45-2.52(1 \mathrm{H}, \mathrm{m}), 3.33-3.42(1 \mathrm{H}$, sept, $J=7.06 \mathrm{~Hz}), 3.65(3 \mathrm{H}, \mathrm{s}), 3.91(3 \mathrm{H}, \mathrm{s}), 4.39-4.4(1 \mathrm{H}, \mathrm{m}), 5.06(1 \mathrm{H}, \mathrm{s})$ $6.53-6.62(2 \mathrm{H}, \mathrm{m}), 7.06(1 \mathrm{H}, \mathrm{s}), 7.85(1 \mathrm{H}, \mathrm{s}) .{ }^{13} \mathrm{C}-\mathrm{NMR}(101 \mathrm{MHz}$, $\left.\mathrm{CDCl}_{3}\right): \delta-4.95,-4.15,13.61,18.26,23.15,23.17,25.92$ (3C), 33.37 $46.83,50.89,60.51,74.43,96.18,114.89,115.13,123.28,134.34,148.76$ 154.43, 162.71, 165.87, 173.72, 178.63. FAB-HR-MS $(\mathrm{m} / \mathrm{z})$ : Calcd for $\mathrm{C}_{25} \mathrm{H}_{39} \mathrm{~N}_{2} \mathrm{O}_{4} \mathrm{~S}_{2} \mathrm{Si}: 523.2121(\mathrm{M}+\mathrm{H})^{+}$. Found: 523.2121

Cystothiazole C (1) To a solution of $\mathbf{1 5}(64 \mathrm{mg}, 0.122 \mathrm{mmol})$ in THF $(1.5 \mathrm{ml})$ was added $1.0 \mathrm{M}$ solution of tetrabutylammonium fluoride in THF $(0.61 \mathrm{mmol})$ at $0{ }^{\circ} \mathrm{C}$. After being stirred for $3 \mathrm{~h}, \mathrm{H}_{2} \mathrm{O}$ was added. The mixture was diluted and extracted with EtOAc. The organic layer was washed with brine, dried $\left(\mathrm{Na}_{2} \mathrm{SO}_{4}\right)$, filtered and concentrated in vacuo. The residue was purified by flash silica gel column chromatography $(1 \mathrm{~g}, n-$ hexane/EtOAc $=2 / 1)$ to afford cyctothiazole $\mathrm{C}(1,30 \mathrm{mg}, 60 \%)$ as a colorless oil. 1: $[\alpha]_{\mathrm{D}}^{21}+142.7^{\circ}\left(c=1.25, \mathrm{CHCl}_{3}\right)$, IR (neat): $3423,3109,1708,1620$, $1147 \mathrm{~cm}^{-1},{ }^{1} \mathrm{H}-\mathrm{NMR}\left(400 \mathrm{MHz}, \mathrm{CDCl}_{3}\right): \delta 1.18(3 \mathrm{H}, \mathrm{d}, J=7.0 \mathrm{~Hz}), 1.44$ $(6 \mathrm{H}, \mathrm{d}, J=6.6 \mathrm{~Hz}), 2.93(1 \mathrm{H}$, br. s), $3.37(1 \mathrm{H}$, sept, $J=6.6 \mathrm{~Hz}), 3.65(3 \mathrm{H}, \mathrm{s})$, $3.70(3 \mathrm{H}, \mathrm{s}), 4.17(1 \mathrm{H}, \mathrm{dq}, J=7.0,4.5 \mathrm{~Hz}), 4.52(1 \mathrm{H}, \mathrm{dd}, J=4.5,4.5 \mathrm{~Hz})$, $5.09(1 \mathrm{H}, \mathrm{s}), 6.61(1 \mathrm{H}, \mathrm{dd}, J=15.9,5.0 \mathrm{~Hz}), 6.68(1 \mathrm{H}, \mathrm{d}, J=15.9 \mathrm{~Hz}), 7.06$ $(1 \mathrm{H}, \mathrm{s}), 7.85(1 \mathrm{H}, \mathrm{s}) .{ }^{13} \mathrm{C}-\mathrm{NMR}\left(101 \mathrm{MHz}, \mathrm{CDCl}_{3}\right): \delta 12.48,23.15(2 \mathrm{C})$, $33.35,40.47,51.13,55.68,74.79,91.54,114.77,115.15,123.52,132.71$, 148.82, 154.63, 162.55, 168.67, 176.90, 178.57. FAB-HR-MS (m/z): Calcd for $\mathrm{C}_{19} \mathrm{H}_{25} \mathrm{~N}_{2} \mathrm{O}_{4} \mathrm{~S}_{2}: 409.1256(\mathrm{M}+\mathrm{H})^{+}$. Found: 409.1258

Cystothiazole A (2) To a solution of cystothiazole C $(\mathbf{1}, 9.3 \mathrm{mg}$, $0.023 \mathrm{mmol})$ in $\mathrm{CH}_{2} \mathrm{Cl}_{2}(0.5 \mathrm{ml})$ were added $\mathrm{Me}_{3} \mathrm{O}^{+} \mathrm{BF}_{4}^{-}(10 \mathrm{mg}$, $0.067 \mathrm{mmol})$ and protone-sponge $(14 \mathrm{mg}, 0.065 \mathrm{mmol})$ at $0{ }^{\circ} \mathrm{C}$. After stirring for $1.5 \mathrm{~h}$ at $0{ }^{\circ} \mathrm{C}$, additional $\mathrm{Me}_{3} \mathrm{O}^{+} \mathrm{BF}_{4}^{-}(10 \mathrm{mg}, 0.067 \mathrm{mmol})$ and protone sponge (14 mg, $0.0653 \mathrm{mmol}$ ) was added. The mixture was warmed to room temperature and stirred for $1 \mathrm{~h}$. The reaction was quenched with $\mathrm{H}_{2} \mathrm{O}$. The separated organic layer was washed with brine, dried and concentrated in vacuo. The residue was purified by silica-gel column chromatography $(1 \mathrm{~g}$, $n$-hexane/EtOAc $=5 / 1)$ to afford the starting material $(1,3.4 \mathrm{mg}, 37 \%$ recovery) and cystothiazole $\mathrm{A}(\mathbf{2}, 4 \mathrm{mg}, 35 \%)$ as a colorless oil. $\mathbf{2}:[\alpha]_{\mathrm{D}}^{23}+105.2^{\circ}$ $\left(c=0.34, \mathrm{CHCl}_{3}\right),{ }^{1} \mathrm{H}-\mathrm{NMR}\left(400 \mathrm{MHz}, \mathrm{CDCl}_{3}\right): \delta 1.21(3 \mathrm{H}, \mathrm{d}, J=7.0 \mathrm{~Hz})$ $1.44(6 \mathrm{H}, \mathrm{d}, J=6.8 \mathrm{~Hz}), 3.33(3 \mathrm{H}, \mathrm{s}), 3.37(1 \mathrm{H}, \mathrm{sept}, J=6.8 \mathrm{~Hz}), 3.60(3 \mathrm{H}$ s), $3.66(3 \mathrm{H}, \mathrm{s}), 3.81(1 \mathrm{H}, \mathrm{dd}, J=7.0,7.0 \mathrm{~Hz}), 4.14-4.21(1 \mathrm{H}, \mathrm{m}), 4.97(1 \mathrm{H}$, s), $6.41(1 \mathrm{H}, \mathrm{dd}, J=15.8,7.5 \mathrm{~Hz}), 6.57(1 \mathrm{H}, \mathrm{d}, J=15.8 \mathrm{~Hz}), 7.09(1 \mathrm{H}, \mathrm{s})$, $7.84(1 \mathrm{H}, \mathrm{s}) .{ }^{13} \mathrm{C}-\mathrm{NMR}\left(101 \mathrm{MHz}, \mathrm{CDCl}_{3}\right): \delta 14.11,23.17(2 \mathrm{C}), 33.37$, $39.86,50.81,55.53,57.01,84.41,91.12,114.82,114.99,125.61,131.63$, 148.76, 154.47, 162.58, 167.73, 176.75, 178.63. FAB-HR-MS $(\mathrm{m} / \mathrm{z})$ : Calcd for $\mathrm{C}_{20} \mathrm{H}_{27} \mathrm{~N}_{2} \mathrm{O}_{4} \mathrm{~S}_{2}: 423.1412(\mathrm{M}+\mathrm{H})^{+}$. Found: 423.1413

2-(2'-Isopropenyl[2,4']bithiazolyl-4-methylenethio)benzothiazole (18) To a solution of the reported primary alcohol $\left.17^{25}\right)(0.29 \mathrm{~g}, 1.22 \mathrm{mmol})$ and 2-mercaptobenzothiazole $(0.24 \mathrm{~g}, 1.43 \mathrm{mmol})$ in THF $(6 \mathrm{ml})$ were added triphenylphosphine $(0.45 \mathrm{~g}, 1.72 \mathrm{mmol})$ and $40 \%$ solution of diethylazodicarboxylate in toluene $(0.57 \mathrm{ml})$ at room temperature. After being stirred for $10 \mathrm{~min}$, the mixture was concentrated in vacuo and purified by flash silica gel column chromatography $(20 \mathrm{~g}, n$-hexane/EtOAc $=4 / 1)$ to afford $\mathbf{1 8}$ $(0.40 \mathrm{~g}, 85 \%)$ as colorless needles. 18: $\mathrm{mp} 104-107^{\circ} \mathrm{C}$, IR (KBr): 1425 ,
992, $746 \mathrm{~cm}^{-1},{ }^{1} \mathrm{H}-\mathrm{NMR}\left(400 \mathrm{MHz}, \mathrm{CDCl}_{3}\right): \delta 2.27(3 \mathrm{H}, \mathrm{s}), 4.77(1 \mathrm{H}, \mathrm{s})$, $4.77(1 \mathrm{H}, \mathrm{s}), 5.36(1 \mathrm{H}, \mathrm{br} . \mathrm{s}), 5.90(1 \mathrm{H}, \mathrm{s}), 7.28-7.32(1 \mathrm{H}, \mathrm{m}), 7.35(1 \mathrm{H}, \mathrm{s})$ $7.40-7.45(1 \mathrm{H}, \mathrm{m}), 7.74-7.76(1 \mathrm{H}, \mathrm{m}), 7.86(1 \mathrm{H}, \mathrm{s}), 7.90-7.92(1 \mathrm{H}, \mathrm{m})$ ${ }^{13} \mathrm{C}-\mathrm{NMR}\left(101 \mathrm{MHz}, \mathrm{CDCl}_{3}\right): \delta 20.42,33.10,115.69,117.41,117.73$, $121.04,121.60,124.34,126.06,135.46,137.77,149.35,152.25,153.14$ 163.06, 165.95, 169.89. Anal. Calcd for $\mathrm{C}_{17} \mathrm{H}_{13} \mathrm{~N}_{3} \mathrm{~S}_{4}: \mathrm{C}, 52.68 ; \mathrm{H}, 3.38 ; \mathrm{N}$, 10.84. Found: C, $52.76 ; \mathrm{H}, 3.65 ; \mathrm{N}, 10.66$.

2-(2'-Isopropenyl[2,4']bithiazolyl-4-methylenesulfonyl)benzothiazole (7) To a solution of $17(0.493 \mathrm{~g}, 1.27 \mathrm{mmol})$ in EtOH $(10 \mathrm{ml})$ were added $\mathrm{Mo}_{7} \mathrm{O}_{24}\left(\mathrm{NH}_{4}\right)_{6} \cdot 4 \mathrm{H}_{2} \mathrm{O}(0.157 \mathrm{~g}, 0.127 \mathrm{mmol})$ and $30 \% \mathrm{H}_{2} \mathrm{O}_{2}(2 \mathrm{ml})$. After being stirred for $2 \mathrm{~h}$, additional $\mathrm{Mo}_{7} \mathrm{O}_{24}\left(\mathrm{NH}_{4}\right)_{6} \cdot 4 \mathrm{H}_{2} \mathrm{O}(0.1 \mathrm{~g}, 0.091 \mathrm{mmol})$ and $30 \% \mathrm{H}_{2} \mathrm{O}_{2}(1 \mathrm{ml})$ were added. The mixture was stirred for additional $1 \mathrm{~h}$, and diluted with $\mathrm{H}_{2} \mathrm{O}$ and EtOAc. The organic layer was washed with $10 \%$ aqueous $\mathrm{Na}_{2} \mathrm{~S}_{2} \mathrm{O}_{3}$ and brine, dried $\left(\mathrm{Na}_{2} \mathrm{SO}_{4}\right)$, filtered and concentrated. The residue was purified by flash silica gel column chromatography $(30 \mathrm{~g}, n$ hexane/EtOAc $=2 / 1)$ to afford $7(0.431 \mathrm{~g}, 81 \%)$ as colorless needles. $7: \mathrm{mp}$ $157-160^{\circ} \mathrm{C}$, IR $(\mathrm{KBr}): 1338,1148 \mathrm{~cm}^{-1},{ }^{1} \mathrm{H}-\mathrm{NMR}\left(400 \mathrm{MHz}, \mathrm{CDCl}_{3}\right): \delta$ $2.22-2.23(3 \mathrm{H}, \mathrm{m}), 4.99(2 \mathrm{H}, \mathrm{s}), 5.34(1 \mathrm{H}, \mathrm{br} . \mathrm{s}), 5.86(1 \mathrm{H}, \mathrm{s}), 7.19(1 \mathrm{H}, \mathrm{s})$, $7.37(1 \mathrm{H}, \mathrm{s}), 7.57-7.60(1 \mathrm{H}, \mathrm{m}), 7.63-7.67(1 \mathrm{H}, \mathrm{m}), 7.92-7.94(1 \mathrm{H}, \mathrm{m})$, $8.28-8.30(1 \mathrm{H}, \mathrm{m}) .{ }^{13} \mathrm{C}-\mathrm{NMR}\left(101 \mathrm{MHz}, \mathrm{CDCl}_{3}\right): \delta 20.34,57.10,115.68$, $117.50,121.64,122.27,125.66,127.60,127.98,137.37,137.63,142.79$, 148.73, 152.72, 163.23, 165.16, 169.91. Anal. Calcd for $\mathrm{C}_{17} \mathrm{H}_{13} \mathrm{~N}_{3} \mathrm{O}_{2} \mathrm{~S}_{4}: \mathrm{C}$, 48.67; H, 3.12; N, 10.02. Found: C, 49.05; H, 3.39; N, 9.45. FAB-MS $(\mathrm{m} / \mathrm{z})$ : $420\left(\mathrm{M}^{+}+\mathrm{H}\right)$

$(2 E, 6 E)-(4 R, 5 S)-5$-(tert-Butyldimethylsilyloxy)-7-(2'-isopropenyl[2,4']bithiazolyl-4-yl)-3-methoxy-4-methylhepta-2,6-dienoic Acid Methyl Ester (19) and $(2 E, 6 Z)-(4 R, 5 S)-5$-(tert-Butyldimethylsilyloxy)-7-(2'-isopropenyl-[2,4']bithiazolyl-4-yl)-3-methoxy-4-methylhepta-2,6-dienoic Acid Methyl Ester (20) To a solution of $7(0.172 \mathrm{~g}, 0.410 \mathrm{mmol})$ in THF $(1.5 \mathrm{ml})$ was added $1.06 \mathrm{M}$ solution of lithium bis(trimethylsilylamide) in THF $(0.37 \mathrm{ml}, 0.39 \mathrm{mmol})$ at $-60^{\circ} \mathrm{C}$. After being stirred at $-60{ }^{\circ} \mathrm{C}$ for $0.5 \mathrm{~h}, \mathbf{5 b}(0.118 \mathrm{~g}, 0.373 \mathrm{mmol})$ in THF $(1 \mathrm{ml})$ was slowly added. The mixture was gradually warmed to $-20^{\circ} \mathrm{C}$ and stirred for $0.5 \mathrm{~h}$. The reaction was quenched with saturated aqueous $\mathrm{NH}_{4} \mathrm{Cl}$ at $0^{\circ} \mathrm{C}$ and warmed to room temperature. The mixture was extracted with EtOAc and washed with brine. The organic layer was dried $\left(\mathrm{Na}_{2} \mathrm{SO}_{4}\right)$, filtered and concentrated in vacuo. The residue was purified by flash silica gel column chromatography $(10 \mathrm{~g}, n$ hexane/EtOAc $=8 / 1)$ to afford $(6 Z)-\mathbf{2 0}(4 \mathrm{mg}, 2 \%)$ and $(6 E)-19(98 \mathrm{mg}, 51 \%)$ as colorless oil. $(6 E)-19$ : $[\alpha]_{\mathrm{D}}^{23}+78.4^{\circ}\left(c=1.845, \mathrm{CHCl}_{3}\right)$, IR (neat): 3115 , $1710,1621 \mathrm{~cm}^{-1},{ }^{1} \mathrm{H}-\mathrm{NMR}\left(400 \mathrm{MHz}, \mathrm{CDCl}_{3}\right): \delta 0.018(3 \mathrm{H}, \mathrm{s}), 0.037(3 \mathrm{H}$, s), $0.92(9 \mathrm{H}, \mathrm{s}), 1.16(3 \mathrm{H}, \mathrm{d}, J=6.7 \mathrm{~Hz}), 2.28(3 \mathrm{H}, \mathrm{s}), 3.60(3 \mathrm{H}, \mathrm{s}), 3.65(3 \mathrm{H}$ s), $4.06(1 \mathrm{H}, \mathrm{m}), 4.41(1 \mathrm{H}, \mathrm{dd}, J=6.7,4.8 \mathrm{~Hz}), 4.96(1 \mathrm{H}, \mathrm{s}), 5.35(1 \mathrm{H}, \mathrm{bs})$, $5.91(1 \mathrm{H}, \mathrm{s}), 6.50-6.59(2 \mathrm{H}, \mathrm{m}), 7.06(1 \mathrm{H}, \mathrm{s}), 7.89(1 \mathrm{H}, \mathrm{s}) .{ }^{13} \mathrm{C}-\mathrm{NMR}$ $\left(101 \mathrm{MHz}, \mathrm{CDCl}_{3}\right): \delta-4.97,-4.08,13.10,18.18,20.44,25.86$ (3C), 41.67, $50.72,55.37,75.55,90.60,114.72,115.48,117.22,122.89,134.95,137.84$ 149.76, 154.89, 162.25, 167.83, 169.70, 177.70. FAB-HR-MS $(\mathrm{m} / \mathrm{z})$ : Calcd for $\mathrm{C}_{25} \mathrm{H}_{37} \mathrm{~N}_{2} \mathrm{O}_{4} \mathrm{~S}_{2} \mathrm{Si}: 521.1964(\mathrm{M}+\mathrm{H})^{+}$. Found: 521.1965. (6Z)-20: ${ }^{1} \mathrm{H}-$ NMR (400 MHz, CDCl $): \delta 0.034(3 \mathrm{H}, \mathrm{s}), 0.063(3 \mathrm{H}, \mathrm{s}), 0.93(9 \mathrm{H}, \mathrm{s}), 1.15$ $(3 \mathrm{H}, \mathrm{d}, J=7.0 \mathrm{~Hz}), 2.28(3 \mathrm{H}, \mathrm{s}), 2.46-2.52(1 \mathrm{H}, \mathrm{m}), 3.65(3 \mathrm{H}, \mathrm{s}), 3.92(3 \mathrm{H}$ s), $4.41(1 \mathrm{H}, \mathrm{dd}, J=4.5,4.5 \mathrm{~Hz}), 5.06(1 \mathrm{H}, \mathrm{s}), 5.36(1 \mathrm{H}, \mathrm{s}), 5.91(1 \mathrm{H}, \mathrm{s})$ $6.54-6.62(2 \mathrm{H}, \mathrm{m}), 7.07(1 \mathrm{H}, \mathrm{s}), 7.90(1 \mathrm{H}, \mathrm{s}) .{ }^{13} \mathrm{C}-\mathrm{NMR}(101 \mathrm{MHz}$, $\left.\mathrm{CDCl}_{3}\right): \delta-4.95,-4.07,13.12,18.20,20.45,25.87$ (3C), 41.68, 50.74 $55.38,75.56,90.61,114.74,115.50,117.24,122.91,134.96,137.85$ 149.78, 154.91, 162.26, 167.85, 169.72, 177.72. FAB-HR-MS (m/z): Calcd for $\mathrm{C}_{25} \mathrm{H}_{37} \mathrm{~N}_{2} \mathrm{O}_{4} \mathrm{~S}_{2} \mathrm{Si}: 521.1964(\mathrm{M}+\mathrm{H})^{+}$. Found: 521.1965 .

Cystothiazole D (3) To a solution of $\mathbf{1 9}(15 \mathrm{mg}, 0.0288 \mathrm{mmol})$ in THF $(1 \mathrm{ml})$ was added $1.0 \mathrm{M}$ solution of tetrabutylammonium fluoride in THF $(0.086 \mathrm{ml}, 0.086 \mathrm{mmol})$ at $0{ }^{\circ} \mathrm{C}$. After being stirred for $3 \mathrm{~h}, \mathrm{H}_{2} \mathrm{O}$ was added The mixture was diluted and extracted with EtOAc. The organic layer was washed with brine, dried $\left(\mathrm{Na}_{2} \mathrm{SO}_{4}\right)$, filtered and concentrated in vacuo. The residue was purified by flash silica gel column chromatography $(1 \mathrm{~g}, n$ hexane $/ \mathrm{EtOAc}=2 / 1)$ to afford $3(6.2 \mathrm{mg}, 53 \%)$ as a colorless oil. $3:[\alpha]_{D}^{24}$ $+136.4^{\circ}\left(c=0.84, \mathrm{CHCl}_{3}\right)$, IR (neat): $3424,3110,1708,1621,1147 \mathrm{~cm}^{-1}$ ${ }^{1} \mathrm{H}-\mathrm{NMR}\left(400 \mathrm{MHz}, \mathrm{CDCl}_{3}\right): \delta 1.18(3 \mathrm{H}, \mathrm{d}, J=7.1 \mathrm{~Hz}), 2.28(3 \mathrm{H}, \mathrm{s}), 2.91$ $(1 \mathrm{H}, \mathrm{d}, J=2.8 \mathrm{~Hz}), 3.66(3 \mathrm{H}, \mathrm{s}), 3.70(3 \mathrm{H}, \mathrm{s}), 4.18(1 \mathrm{H}, \mathrm{dq}, J=7.1,4.5 \mathrm{~Hz})$, $4.50-4.54(1 \mathrm{H}, \mathrm{m}), 5.09(1 \mathrm{H}, \mathrm{s}), 5.36(1 \mathrm{H}, \mathrm{d}, J=1.0 \mathrm{~Hz}), 5.91(1 \mathrm{H}, \mathrm{s}), 6.62$ $(1 \mathrm{H}, \mathrm{dd}, J=15.9,5.0 \mathrm{~Hz}), 6.68(1 \mathrm{H}, \mathrm{d}, J=15.9 \mathrm{~Hz}), 7.07(1 \mathrm{H}, \mathrm{s}), 7.90$ $(1 \mathrm{H}, \mathrm{s}) .{ }^{13} \mathrm{C}-\mathrm{NMR}\left(101 \mathrm{MHz}, \mathrm{CDCl}_{3}\right): \delta 12.46,20.45,40.47,51.13,55.69$ $74.79,91.56,115.42,115.56,117.28,123.48,132.77,137.84,149.74$ $154.67,162.42,168.68,169.78,176.91$. FAB-HR-MS $(\mathrm{m} / \mathrm{z})$ : Calcd for $\mathrm{C}_{10} \mathrm{H}_{23} \mathrm{~N}_{2} \mathrm{O}_{4} \mathrm{~S}_{2}: 407.1099(\mathrm{M}+\mathrm{H})^{+}$. Found: 407.1099.

Melithiazol B (4) To a solution of $3(11.3 \mathrm{mg}, 0.027 \mathrm{mmol})$ in $\mathrm{CH}_{2} \mathrm{Cl}_{2}$ $(0.7 \mathrm{ml})$ were added $\mathrm{Me}_{3} \mathrm{O}^{+} \mathrm{BF}_{4}^{-}(12.3 \mathrm{mg}, 0.083 \mathrm{mmol})$ and protone sponge 
( $17.9 \mathrm{mg}, 0.083 \mathrm{mmol}$ ) at $0{ }^{\circ} \mathrm{C}$. After stirring for $3 \mathrm{~h}, \mathrm{H}_{2} \mathrm{O}$ was added. The separated organic layer was extracted with EtOAc, washed with brine, dried and concentrated in vacuo. The residue was purified by silica-gel column chromatography $(1 \mathrm{~g}, n$-hexane/EtOAc $=4 / 1)$ to afford $4(7.2 \mathrm{mg}, 62 \%)$ and the starting material $3\left(1.4 \mathrm{mg}, 12 \%\right.$ recovery) as a colorless oil. 4 : $[\alpha]_{\mathrm{D}}^{24}$ $+94.0^{\circ}\left(c=0.72, \mathrm{CHCl}_{3}\right),{ }^{1} \mathrm{H}-\mathrm{NMR}\left(400 \mathrm{MHz}, \mathrm{CDCl}_{3}\right): \delta 1.22(3 \mathrm{H}, \mathrm{d}$, $J=7.1 \mathrm{~Hz}), 2.28(3 \mathrm{H}, \mathrm{s}), 3.34(3 \mathrm{H}, \mathrm{s}), 3.61(3 \mathrm{H}, \mathrm{s}), 3.67(3 \mathrm{H}, \mathrm{s}), 3.82(1 \mathrm{H}$, dd, $J=7.8,7.8 \mathrm{~Hz}), 4.14-4.21(1 \mathrm{H}, \mathrm{m}), 4.97(1 \mathrm{H}, \mathrm{s}), 5.37(1 \mathrm{H}, \mathrm{s}), 5.91(1 \mathrm{H}$, s), $6.42(1 \mathrm{H}, \mathrm{dd}, J=15.9,7.6 \mathrm{~Hz}), 6.58(1 \mathrm{H}, \mathrm{d}, J=15.9 \mathrm{~Hz}), 7.10(1 \mathrm{H}, \mathrm{s})$, $7.89(1 \mathrm{H}, \mathrm{s}) .{ }^{13} \mathrm{C}-\mathrm{NMR}\left(101 \mathrm{MHz}, \mathrm{CDCl}_{3}\right): \delta 14.09,20.44,39.86,50.81$, $55.53,57.02,84.39,91.11,115.26,115.59,117.31,125.53,131.82,137.82$, 149.65, 154.48, 162.44, 167.73, 169.80, 176.75. FAB-HR-MS $(\mathrm{m} / \mathrm{z})$ : Calcd for $\mathrm{C}_{20} \mathrm{H}_{25} \mathrm{~N}_{2} \mathrm{O}_{4} \mathrm{~S}_{2}: 421.1256(\mathrm{M}+\mathrm{H})^{+}$. Found: 421.1257

Acknowledgement The authors are grateful to Professor Noritaka Chida, Department of Applied Chemistry, Keio University, for cooperation in FAB-HR-MS measurement.

\section{References and Notes}

1) Ojika M., Suzuki Y., Tsukamoto A., Sakagami Y., Fudou R., Yoshimura T., Yamanaka S., J. Antibiot., 51, 275-281 (1998).

2) Suzuki Y., Ojika M., Sakagami Y., Fudou R., Yamanaka S., Tetrahedron, 54, 11399-11404 (1998).

3) Böhlendrof B., Herrmann M., Hecht H.-J., Sasse F., Forche E., Kunze B., Reichenbach H., Höfle G., Eur. J. Org. Chem., 1999, 2601-2608 (1999).

4) Gerth K., Irschik H., Reichenbach H., Trowitzsch W., J. Antibiot., 33, $1474-1479$ (1980).

5) Trowitzsch W., Reifenstahl G., Wray V., Gerth K., J. Antibiot., 33, $1480-1490$ (1980).

6) Trowitzsch W., Höfle G., Sheldrick W. S., Tetrahedron Lett., 22, 3829-3832 (1981).

7) Thierbach G., Reichenbach H., Biochim. Biophys. Acta, 638, 282-
289 (1981).

8) Ojika M., Watanabe T., Qi J., Tanino T., Sakagami Y., Tetrahedron, 60, 187-194 (2004).

9) Williams D. R., Patnaik S., Clark M. C., J. Org. Chem., 66, 84638469 (2001).

10) Kato K., Nishimura A., Yamamoto Y., Akita H., Tetrahedron Lett., 43, 643-645 (2002).

11) Kato K., Sasaki T., Takayama H., Akita H., Tetrahedron, 59, 2679 2685 (2003).

12) Sasaki T., Kato K., Akita H., Chem. Pharm. Bull., 52, 770-771 (2004).

13) Akita H., Sutou N., Sasaki T., Kato K., Tetrahedron, 62, 1159211598 (2006).

14) Bach T., Heuser S., Chem. Eur. J., 8, 5585-5592 (2002).

15) DeRoy P. L., Charette A. B., Org. Lett., 5, 4163-4165 (2003).

16) Shao J., Panek J. S., Org. Lett., 6, 3083-3085 (2004).

17) Bach T., Heuser S., Angew. Chem. Int. Ed., 40, 3184-3185 (2001).

18) Takayama H., Kato K., Kimura M., Akita H., Heterocycles, 71, 7585 (2007).

19) Blakemore P. R., J. Chem. Soc., Perkin Trans. I, 2002, 2563-2585 (2002).

20) Akita H., Kawaguchi T., Enoki Y., Oishi T., Chem. Pharm. Bull., 38, 323 -328 (1990).

21) Akita H., Todoroki R., Endo H., Ikari Y., Oishi T., Synthesis, 1993, $513-516$ (1993).

22) Kato K., Ono M., Akita H., Tetrahedron Asymmetry, 8, 2295-2298 (1997).

23) Osaka Yuki Kagaku Kogyo Co., Ltd. (Japan), Japan Kokai Tokkyo Koho JP 5-276966 (1993).

24) Inanaga J., Baba Y., Hanamoto T., Chem. Lett., 1993, 241-244 (1993).

25) Akita H., Sasaki T., Takayama H., Kato K., Heterocycles, 66, 219 228 (2005) 